\title{
FORTALECIMIENTO Y DESARROLLO INTEGRAL DE LOS COLABORADORES DE GESTIÓN ADMINISTRATIVA DE LA UNIVERSIDAD CATÓLICA DE SANTIAGO DE GUAYAQUIL
}

\author{
STRENGTHENING AND INTEGRAL DEVELOPMENT OF EMPLOYEES OF \\ ADMINISTRATIVE MANAGEMENT OF THE CATHOLIC UNIVERSITY OF \\ SANTIAGO DE GUAYAQUIL
}

\author{
ADELA ZURITA FABRE ${ }^{1}$, MARÍA PAULINA CHING CORREA², NELLY DÍAZ AVEIGA³ ${ }^{3}$ EUGENIO CHALÉN YÉPEZ4, \\ LUIS PAZMIÑO CRESPO ${ }^{5}$, DIEGO PAZMIÑO CRESPO 6

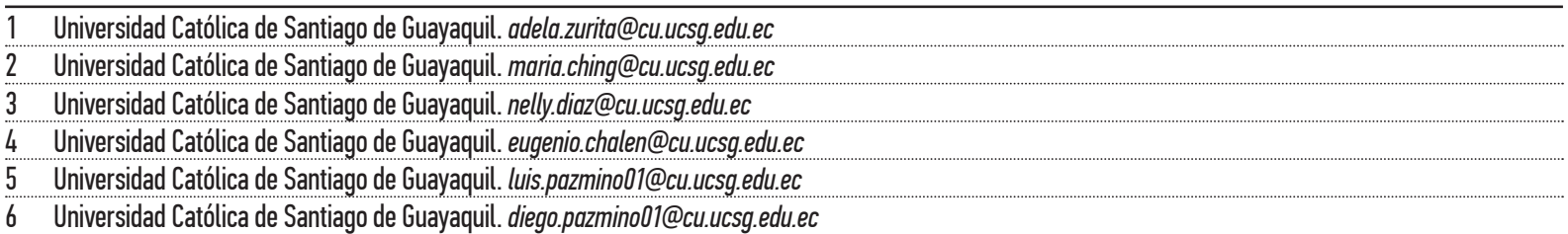

RESUMEN

El presente artículo es el resultado de una investigación que se planteó como objetivo elaborar un diagnóstico de necesidades de capacitación orientado al fortalecimiento y desarrollo integral de colaboradores de gestión administrativa de la Universidad Católica de Santiago de Guayaquil (UCSG). Se partió de un enfoque cuantitativo y descriptivo con la participación de 248 personas seleccionadas mediante un muestreo estratificado, de una población total de 686 colaboradores de gestión administrativa, entre funcionarios y personal de apoyo, sin especificidad en género, antigüedad o nivel jerárquico. Los resultados resaltan que los encuestados están conscientes de que la actualización y/o adquisición de conocimientos son básicas en su crecimiento profesional y personal y en su eficiente desempeño. De igual forma, consideran que los temas a ser incluidos en el programa de capacitación deben responder a necesidades laborales de la institución en general y de los cargos en particular, reconociendo que estos, incentivan al colaborador en el desarrollo de la innovación y la creatividad, base para la solución de conflictos y la toma de decisiones. Con base a lo anterior, se presenta el diagnóstico de necesidades de capacitación que podría ser incluido en los programas a ser diseñados por la institución.

PALABRAS CLAVE: diagnóstico de necesidades, desarrollo integral, programa de capacitación, ventaja competitiva institucional, beneficios de la capacitación.
This article is the result of an investigation that was raised as a goal to elaborate a diagnosis of training needs geared to the strengthening and development of employees of administrative management of the Catholic University of Santiago de Guayaquil (UCSG). Is split of an approach quantitative and descriptive with the participation of 248 people selected through a sampling stratified, of a population total of 686 contributors of management administrative, between officials and personal of support, without specificity in gender, seniority or level hierarchical. The results highlight that the respondents are aware that updating and/or acquisition of knowledge are basic in their professional and personal growth and its efficient performance. Similarly, they believe that the topics to be included in the training programme must respond to labour needs of the institution in general and jobs in particular, recognizing that these incentives to collaborating in the development of innovation and creativity, base for conflict resolution and decision-making. Based on the above, is the diagnosis of training needs that could be included in programs to be designed by the institution

KEYWORDS: diagnosis of needs, developing comprehensive, program of training, advantage competitive institutional, benefits of the training. 
INTRODUCCIÓN

El quehacer cotidiano de las empresas involucra la presencia de personas, que hacen que los diferentes departamentos funcionen acorde con los objetivos previamente determinados. Una forma de optimizar los resultados de esas personas es a través de programas de capacitación bien diseñados, ajustados a la realidad institucional y dentro de los parámetros de excelencia y calidad. Programas a través de los cuales, no solamente se busque la profesionalización y/o experticia del colaborador en cuanto a sus funciones en general, sino que también se constituya en un proceso de motivación implícita, orientado a establecer y mantener un alto nivel de compromiso y sentido de pertenencia (Chiang, Núñez, Martín, y Salazar, 2010) hacia la empresa de la que forman parte.

Las empresas, independientemente de su tamaño, actividad o tipo de capital, tienen una estructura que facilita la coordinación y comunicación interna, entre departamentos, entre personas, y entre éstas y los clientes. Si bien cada departamento o sección tiene sus propios procesos y objetivos, no es menos cierto que el resultado de esa área afecta o influye en el resultado que debe presentar otra área o departamento, y así sucesivamente el ciclo productivo se completa, amparado en la misión, visión y objetivos organizacionales. Por ello, se requiere contar con personas debidamente capacitadas, tanto en lo personal como en lo laboral y profesional, de manera que la empresa pueda participar en un mercado tan competitivo como lo es el actual (Ibarra Almada, 2000).

Las Instituciones de Educación Superior (IES), y concretamente la Universidad Católica de Santiago de Guayaquil (UCSG), no pueden abstraerse de este entorno. Su estructura, fines y ámbito de acción tienen relación con personas, procesos, servicios y comunidad; con una orientación hacia la calidad de la educación superior que se logra partiendo de una cadena de valor claramente identificada, en donde las actividades de gestión administrativa dan el soporte necesario para que se cumplan las funciones sustantivas propias de las universidades.

Por tanto, se hace necesario identificar las necesidades de fortalecimiento y desarrollo integral de los colaboradores de gestión administrativa, es decir, de todas aquellas personas que forman parte del equipo de trabajo en las diferentes unidades departamentales de la UCSG, con funciones claramente definidas, de manera que faciliten una atención ágil, oportuna y pertinente tanto al cliente interno como al externo. Todo ello posibilitará también, establecer parámetros administrativos y técnicos para diseñar e implementar un sistema de capacitación continua que busque potenciar el capital intelectual, cuestión que constituye el centro de atención del presente artículo.

Se aborda en este trabajo, un estudio realizado sobre el proceso interno de capacitación y desarrollo en la UCSG, a fin de conocer las experiencias y percepciones de los colaboradores de la institución, que facilite la elaboración de un diagnóstico de necesidades. A tales fines, se aplicó una encuesta cuyos resultados, analizados mediante un software estadístico, permitieron elaborar un diagnóstico de necesidades en función de competencias laborales y profesionales. Todo este proceso fue desarrollado mediante una investigación con enfoque cuantitativo-descriptivo, en el que participaron 248 personas seleccionadas, mediante un muestreo estratificado, partiendo de una población de 686 colaboradores de gestión administrativa.

Se pudo apreciar entre los participantes, aceptación ante un programa continuo de capacitación que contemple temas generales relacionados con el quehacer institucional y el desarrollo del sentido de pertenencia, de conjunto con temas relativos a su área de trabajo y otros orientados hacia su desarrollo personal.

Como resultado de esta investigación se lograron identificar las necesidades de capacitación orientadas a desarrollar, en el colaborador de gestión administrativa, las competencias necesarias para cumplir con sus funciones y procesos; de forma tal, que mejore su nivel de satisfacción y motivación con respecto a la tarea.

TENDENCIAS EN TORNO A CAPACITACIÓN Y DESARROLLO Todas las organizaciones funcionan con diferentes sistemas: tecnología, recursos físicos y financieros, infraestructura, capital, procesos, etc.; pero nada de ello es factible si no cuenta con personas que hagan que dichos sistemas se encaminen hacia los objetivos institucionales. La capacidad de desarrollar a esas personas es lo que permite conformar un equipo humano consolidado, comprometido y orientado hacia fines comunes, que haga frente a cambios generados por los avances de la tecnología y la globalización de los mercados, que obligan a las empresas a actualizar sus sistemas para enfrentar las turbulencias e incertidumbres (Chiavenato, 2009). Pero, otra vez, los sistemas no se optimizan solos, lo hacen las personas y es 
a esas personas a las que se debe potenciar en su intelecto y su desempeño, dado que la capacitación es uno de los componentes principales de la gestión exitosa de una institución.

Las personas representan un elemento inteligente con alto potencial de desarrollo y habilidades para adquirir nuevos conocimientos y conceptos, innovar, crear y desarrollar competencias. Si el ser humano reconoce que los nuevos aprendizajes le producirán una recompensa o le generarán algún efecto positivo, entonces orientará sus esfuerzos para repetir la experiencia. Este proceso implica la ley del estímulo y de los incentivos que hace repetitiva la conducta; en este caso, de aprender y recibir los beneficios de ese aprendizaje y que se refleje además en el desempeño (Dolan, Valle Cabrera, Jackson, y Schuler, 2007).

Por otro lado, se debe enfatizar en ciertas especificaciones tales como educación, formación, desarrollo y capacitación. La educación profesional, institucionalizada o no, prepara a las personas para su vida profesional en general; la formación profesional, prepara a la persona para el ejercicio de su función en algún sector del mercado laboral; el desarrollo profesional es el que busca perfeccionar a las personas para ejercer una especialidad en el ámbito de una profesión; y, finalmente, la capacitación es la adaptación sistemática y organizada de la educación, la formación y el desarrollo profesional para la construcción de conocimiento (Chiavenato, 2011) aplicado en un puesto o función dentro de una empresa. Adicionalmente, construir conocimiento significa aprovechar las ideas y experiencias de los colaboradores para transformar, crear, movilizar esfuerzos e innovar, involucrándolos en una auto renovación personal y organizacional (Nonaka, 2007). En este proceso, el conocimiento se vuelve formal y sistemático (explícito) pero a la vez, surge también de un tipo de destreza informal (tácito) que se va desarrollando a medida que se realizan las tareas y el colaborador utiliza su iniciativa para ir mejorando cada vez, generando conocimiento y desarrollando competencias para ponerlas al servicio de la institución y de los usuarios (Gil López y Carrillo Gamboa, 2013).

Si bien el conocimiento es la base del desempeño, la competencia se refiere a su aplicabilidad para un desempeño exitoso y a la vez, está relacionada, al decir de Alles (2009) con características de la personalidad que se reflejan en el comportamiento. Por supuesto, esta relación de conocimiento y competencia puede ser analizada desde dos ámbitos (Blanco Fernández, 2009): en el campo laboral (capacidad para aplicar reglas, procedimientos, instrumentos y desarrollar las destrezas necesarias para atender situaciones en el trabajo) y en el ámbito personal (lo que las personas deben y pueden aprender y desarrollar para su desempeño personal y profesional).

Las competencias se manifiestan en el desempeño, en el saber, el saber ser, el saber estar y el saber actuar y deben ser adecuadas a las exigencias del entorno y del avance de la tecnología (González Bertolín, 2011). Por tanto, en el contexto laboral debe establecerse una clara diferenciación de los diversos conjuntos de competencias, de manera que se diseñe una guía de capacitación adaptable a las necesidades individuales, del puesto de trabajo y de la institución. Debe reconocerse que, dados los diferentes estilos de aprendizaje de las personas, es lógico esperar que la nueva información a recibir, tenga diversos niveles de reconocimiento, codificación, decodificación y puesta en práctica para su propia superación y de la institución (Gallego y Gil, 2012).

\section{CAPACITACIÓN COMO PROCESO Y VENTAJA COMPETITIVA}

Si bien la capacitación es un proceso que se da a corto plazo, mediante el cual las personas actualizan o adquieren nuevos conocimientos y desarrollan o especializan habilidades y competencias (Chiavenato, 2011), se deben esperar también, aprendizajes a largo plazo, dependiendo del nivel de motivación que tengan las personas que participan en estos programas. Debe pretenderse siempre, que exista un equilibrio entre las competencias y el puesto.

La capacitación, como todo proceso, está compuesto por etapas claramente definidas (Chiavenato, 2011; Robbins y Coulter, 2014; Schermerhorn, 2010):

- Diagnóstico: parte de un análisis organizacional que incluye los lineamientos institucionales y un sistema de inducción y reinducción. Un análisis de las personas, en términos de las competencias genéricas y específicas que poseen y los requerimientos del puesto, así como para actualización de procesos o equipos; y el análisis de la evaluación del desempeño;

- Diseño: incluye los objetivos esperados y en función de ellos el contenido a desarrollar, el sector participante, los 
recursos (tecnología, presupuesto y otros elementos logísticos) y los facilitadores;

- Ejecución: contempla la modalidad (presencial o semipresencial) y el seguimiento (control de asistencia, pruebas de conocimiento, etc.);

- Evaluación de resultados: relacionado con una comparación de antes y después en comportamientos y procesos; así como cambios en los resultados logrados con los nuevos aprendizajes, además de la revisión del costo-beneficio del programa.

En términos generales la capacitación se convierte en un elemento de ventaja competitiva para la institución, tanto en lo productivo (reducción de costos, aumento de la productividad), en lo es tructural (mejora la calidad, desarrolla identidad y compromiso hacia la institución), como en lo humano (potencia las capacidades en lo técnico y en lo personal, y mejora el desempeño).

\section{METODOLOGÍA}

La investigación realizada tuvo un enfoque cuantitativo-descriptivo, dado que a través de la experiencia y percepción de los colaboradores de gestión administrativa en cuanto al proceso interno de capacitación y desarrollo, se buscó identificar las necesidades de fortalecimiento y desarrollo integral orientadas a proporcionar una atención ágil, oportuna y pertinente tanto al cliente interno como al externo.

Para obtener la información, se procedió en primera instancia a identificar los participantes del proceso. Luego fueron determinados los componentes a ser incluidos en el instrumento para obtener información válida, pasando a tabular los datos y obtener los resultados que permitieron llegar a conclusiones lógicas.

\section{PARTICIPANTES}

De un total de 686 colaboradores, entre funcionarios y personal de apoyo, luego de aplicar la fórmula para poblaciones finitas, con un $95 \%$ de confianza y un margen de error del $5 \%$, se obtuvo una muestra de 248 personas. La selección de la muestra fue estratificada, buscando la participación de colaboradores de diferentes departamentos, sin especificidad en género, antigüedad o nivel jerárquico.

INSTRUMENTOS

En el formato de encuesta utilizado, además de los aspectos personales y laborales, se incluyeron dos secciones relacionadas con capa- citación. La primera, fue una lista de temas de manejo amplio y general para cualquier cargo, con la intención de conocer en cuál de ellos ha participado el entrevistado con la modalidad de curso, seminario o taller. En la segunda sección, fueron abordados aspectos relacionados con la importancia de un programa de capacitación en la UCSG, con opciones de respuestas basadas en la escala de Likert.

Como fue mencionado anteriormente, la efectividad de la capacitación depende no solo del tema, instructor, etc., sino también del interés del participante y su nivel de motivación hacia un mejoramiento en el plano laboral y profesional. Con esta consideración, se buscó conocer qué tan importante es para el encuestado su capacitación; cómo debería estar conformado un programa de capacitación; y el origen de los temas a incluir en dicho programa.

RESULTADOS

La conformación de la muestra según aspectos personales y laborales se presenta en la Tabla 1.

TABLA 1. CLASIFICACIÓN DE ENCUESTAdos SEGÚN GÉNERO, EdAD Y FORMACIÓN ACADÉMICA

\begin{tabular}{|c|c|c|c|c|}
\hline \multicolumn{2}{|c|}{ ASPECTOS } & DESCRIPCIÓN & NÚMERO & PORCENTAJE \\
\hline \multirow{6}{*}{ 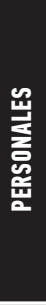 } & \multirow{2}{*}{ GÉNERO } & Femenino & 168 & 67,74 \\
\hline & & Masculino & 80 & 32,26 \\
\hline & \multirow{4}{*}{ EDAD } & Hasta 20 años & 5 & 2,02 \\
\hline & & 21 a 25 años & 29 & 11,69 \\
\hline & & 26 a 30 años & 44 & 17,74 \\
\hline & & Más de 31 años & 170 & 68,55 \\
\hline \multirow{14}{*}{$\begin{array}{l}\text { 岂 } \\
\text { 总 } \\
\text { 总 }\end{array}$} & \multirow{2}{*}{ UNIDAD } & Académica & 76 & 30,65 \\
\hline & & Administrativa & 172 & 69,35 \\
\hline & \multirow{8}{*}{ ANTIGİEDAD } & Menos de 6 meses & 7 & 2,82 \\
\hline & & $\begin{array}{l}\text { De } 6 \text { meses a } \\
\text { menos de } 1 \text { año }\end{array}$ & 9 & 3,63 \\
\hline & & De 1 a 5 años & 58 & 23,39 \\
\hline & & De 6 a 10 años & 71 & 28,53 \\
\hline & & De 11 a 15 años & 35 & 14,11 \\
\hline & & De 16 a 20 años & 11 & 4,44 \\
\hline & & De 21 a 25 años & 29 & 11,29 \\
\hline & & Más de 25 años & 29 & 11,69 \\
\hline & \multirow{4}{*}{$\begin{array}{l}\text { NIVEL } \\
\text { JERÁRQUICO }\end{array}$} & Directivo & 14 & 5,64 \\
\hline & & $\begin{array}{l}\text { Coordinación, } \\
\text { jefatura, asesoría }\end{array}$ & 34 & 13,71 \\
\hline & & $\begin{array}{l}\text { Personal apoyo } \\
\text { administrativo }\end{array}$ & 189 & 76,21 \\
\hline & & $\begin{array}{l}\text { Personal apoyo } \\
\text { operativo }\end{array}$ & 11 & 4,44 \\
\hline
\end{tabular}


El resultado de las encuestas dio un mayor porcentaje de personal de apoyo administrativo (analistas, asistentes, secretarias, auxiliares de secretaria) con un $76,21 \%$, lo cual fue relevante ya que son las personas que tienen bajo su responsabilidad, la ejecución directa de los procesos y que dan soporte a decisiones de niveles de coordinación y de dirección. En un porcentaje menor $(19,35 \%)$ están representados cargos de niveles altos, de dirección y decisión. Finalmente, un $4,44 \%$ le correspondió al personal de apoyo operativo (intendencia, mensajeros, conserjes, choferes).

El formulario incluyó una lista de cursos, talleres y seminarios a los que pudieron haber asistido los encuestados. Los resultados se presentan en la Tabla 2.

TABLA 2. CURSOS A LOS QUE LOS ENCUESTADOS HAN ASISTIDO

DESCRIPCIÓN NÚMERO

\begin{tabular}{|llc}
\hline B1: & Administración del cambio & 5 \\
\hline B2: & Aplicativos informáticos & 63 \\
\hline B3: & Balance scorecard & 9 \\
\hline B4: & Bases de datos & 16 \\
\hline B5: & Competencias profesionales, laborales, personales & 32 \\
\hline B6: & Comportamiento y comunicación organizacional & 20 \\
\hline B7: & Contabilidad & 26 \\
\hline B8: & Control de calidad & 28 \\
\hline B9: & Creatividad e innovación & 12 \\
\hline B10: & Desarrollo de habilidades del pensamiento & 17 \\
\hline B11: & Desarrollo personal & 35 \\
\hline B12: & Emprendimiento & 9 \\
\hline B13: & Ética profesional e institucional & 8 \\
\hline B14: & Gestión del talento humano & 24 \\
\hline B15: & Gestión y prevención de riesgos & 50 \\
\hline B16: & Idioma extranjero & 17 \\
\hline B17: & Liderazgo & 16 \\
\hline B18: & Manejo de procesos & 20 \\
\hline B19: & Negociación/manejo de conflictos & 9 \\
\hline B20: & Pensamiento crítico & 5 \\
\hline B21: & Planificación estratégica & 12 \\
\hline B22: & Publicidad & 6 \\
\hline B23: & Relaciones públicas & 17 \\
\hline B24: & Salud ocupacional & 37 \\
\hline B25: & Trabajo en equipo & 46 \\
\hline B26: & Ventas & 57 \\
\hline B27: & Desarrollo de aplicaciones informáticas \\
\hline B28: & Ninguno & 27 \\
\hline B29: & Otro & 5 \\
\hline & & 5 \\
\hline
\end{tabular}

Los resultados obtenidos pueden resumirse como sigue: 63 colaboradores asistieron a cursos sobre aplicativos informáticos (B2), 50 a gestión y prevención de riesgos (B15), 46 a trabajo en equipo (B25), 37 a salud ocupacional (B24), y así sucesivamente. Se evidenció la baja participación de los encuestados en los programas que la UCSG diseña para potenciar el desempeño de sus colaboradores. Entre otros cursos que representan algún nivel de importancia para su desempeño, los colaboradores mencionaron:

- Archivología y gestión documentos

- Auditoría en sistemas de información, Sistemas inteligentes

- Bibliotecología, formato Mark 21 Isis Marcy, Z3915, gestión documental normas EadWinisis, encuentro pedagógico del bibliotecario, estándares de calidad en bibliotecas

- Cableado estructurado y fibra óptica

- Coaching, Emd2

- Community Nanogiga

- Evaluación de desempeño

- Gestión de proyectos

- Gestores de la información en la era del conocimiento, Urkund

- Imagen corporativa/personal

- Manejo de la plataforma del IESS, Nómina, Retiro laboral, Reformas laborales

- Metodología de investigación, fortalecimiento de capacidades investigativas

- Presupuesto, Tributación, Finanzas, Compras públicas

- Procesos, productividad y calidad

Como se puede apreciar, muchos de estos temas son específicos de cargos y departamentos que deberán ser tomados en consideración para ser incluidos en futuros programas que diseñe la UCSG.

\section{ASPECTOS NECESARIOS EN EL DISEÑO DE PROGRAMAS DE CAPACITACIÓN}

Se buscó la opinión de los encuestados sobre la necesidad e importancia de la capacitación de los colaboradores de la UCSG. La Tabla 3 resume las respuestas a las siguientes opciones del cuestionario:

1. Aumentar el nivel de conocimientos sobre un tema específico

2. Mejorar el desempeño en el área en que labora

3. Mantener la estabilidad en el puesto 
4. Ascender a un puesto de mayor responsabilidad

5. Obtener un certificado para mejorar la "hoja de vida"

TABLA 3. NECESIDAD E IMPORTANCIA DE LA CAPACITACIÓN

\begin{tabular}{|c|c|c|c|c|c|}
\hline RESPUESTAS & $\begin{array}{l}\text { CONOCI- } \\
\text { MIENTO }\end{array}$ & DESEMPEÑO & ESTABILIDAD & ASCENSO & $\begin{array}{l}\text { HOJA DE } \\
\text { VIDA }\end{array}$ \\
\hline $\begin{array}{l}\text { Acuerdo } \\
\text { parcial }\end{array}$ & 38 & 40 & 108 & 98 & 86 \\
\hline Acuerdo total & 206 & 205 & 75 & 116 & 114 \\
\hline $\begin{array}{l}\text { Desacuerdo } \\
\text { parcial }\end{array}$ & 4 & 3 & 39 & 23 & 31 \\
\hline $\begin{array}{l}\text { Desacuerdo } \\
\text { total }\end{array}$ & 0 & 0 & 26 & 11 & 17 \\
\hline Total & 248 & 248 & 248 & 248 & 248 \\
\hline $\begin{array}{l}\text { Acuerdo } \\
\text { (total y } \\
\text { parcial) }\end{array}$ & $98,39 \%$ & $98,79 \%$ & $73,79 \%$ & $86,29 \%$ & $80,65 \%$ \\
\hline
\end{tabular}

Las respuestas se concentraron (acuerdo parcial y acuerdo total) en el hecho de que la capacitación facilita mayor nivel de conocimiento (244 de 248 encuestados, equivalentes al $98,39 \%$ ) y permite mejorar el nivel de desempeño (245 equivalentes al 98,79\%). Este comportamiento, hace ver que los colaboradores están conscientes de que el refuerzo de conocimientos actuales y la adquisición de otros, es básico en su crecimiento profesional y personal, y que redundará en un desempeño eficiente.

Las opciones de estabilidad en el puesto $(73,79 \%)$, ascenso a cargos de mayor responsabilidad $(86,29 \%)$ y obtener un certificado para la hoja de vida $(80,65 \%)$, aunque no tuvieron un nivel similar de preferencia, fueron también considerados importantes, ya que son complemento de su desarrollo profesional. Por tanto, elevar el nivel de conocimientos para mejorar el desempeño es un indicador que debe ser tomado en consideración cuando se diseñen programas de capacitación en la UCSG.

En cuanto a qué incluir en un plan de capacitación, fueron presentadas las siguientes opciones de respuesta, según se muestra en la Tabla 4:

1. Eventos ocasionales de capacitación sobre temas básicos

2. Eventos regulares y periódicos de capacitación con temas básicos
3. Cursos, seminarios y talleres que induzcan a la innovación y creatividad

4. Cursos, seminarios y talleres que se ajusten a las necesidades laborales

5. Temas de capacitación especializados, según las áreas de trabajo

\section{TABLA 4. PLAN DE CAPACITACIÓN DE LA UCSG}

\begin{tabular}{|c|c|c|c|c|c|}
\hline $\begin{array}{l}\text { RESPUES- } \\
\text { TAS }\end{array}$ & $\begin{array}{l}\text { OCASIONAL. } \\
\text { TEMAS } \\
\text { BÁSICOS }\end{array}$ & $\begin{array}{l}\text { REGULAR. } \\
\text { TEMAS } \\
\text { BÁ́SICOS }\end{array}$ & $\begin{array}{l}\text { INNOVACIÓN } \\
\text { Y CREATIVI- } \\
\text { DAD }\end{array}$ & $\begin{array}{l}\text { MECESIDADES } \\
\text { LABORALES }\end{array}$ & $\begin{array}{l}\text { SEGÚN } \\
\text { ÁREAS } \\
\text { DE } \\
\text { TRABAJO }\end{array}$ \\
\hline $\begin{array}{l}\text { Acuerdo } \\
\text { parcial }\end{array}$ & 111 & 119 & 67 & 43 & 51 \\
\hline $\begin{array}{l}\text { Acuerdo } \\
\text { total }\end{array}$ & 111 & 98 & 167 & 196 & 188 \\
\hline $\begin{array}{l}\text { Desacuerdo } \\
\text { parcial }\end{array}$ & 14 & 21 & 13 & 8 & 7 \\
\hline $\begin{array}{l}\text { Desacuerdo } \\
\text { total }\end{array}$ & 12 & 10 & 1 & 1 & 2 \\
\hline Total & 248 & 248 & 248 & 248 & 248 \\
\hline $\begin{array}{l}\text { Acuerdo } \\
\text { (total y } \\
\text { parcial) }\end{array}$ & $89,52 \%$ & $87,50 \%$ & $94,35 \%$ & $96,37 \%$ & $96,37 \%$ \\
\hline
\end{tabular}

De acuerdo con las respuestas proporcionadas, tal como se aprecia en la Tabla 4, existe una tendencia a estar de acuerdo total o parcial) sobre todas las opciones presentadas. Esto significa, que se espera que el plan de capacitación incluya diferentes temas (básicos y específicos), que respondan a las necesidades laborales de la institución en general y de los cargos en particular. De igual forma, se espera que se les incentive en el desarrollo de la innovación y la creatividad, base para la solución de conflictos y la toma de decisiones.

Al preguntar cómo debía conformarse un plan de capacitación, la Tabla 5 presenta las respuestas a las cinco opciones propuestas:

1. Petición específica de un directivo, funcionario o colaborador;

2. Identificación de necesidades para reforzar un resultado esperado;

3. Diagnóstico de necesidades de capacitación a nivel institucional;

4. Cambios en procesos o en el uso de equipos o aplicativos informáticos;

5. Decisiones centrales del área de desarrollo humano. 
TABLA 5. PREFERENCIA SOBRE LOS TEMAS

\begin{tabular}{|c|c|c|c|c|c|}
\hline RESPUESTAS & $\begin{array}{l}\text { PETICIÓN DE } \\
\text { INTERESADO }\end{array}$ & $\begin{array}{l}\text { REFUERZO DE } \\
\text { RESULTADOS }\end{array}$ & $\begin{array}{l}\text { DIAGNÓSTICO } \\
\text { MECESIDADES }\end{array}$ & $\begin{array}{l}\text { CAMBIOS } \\
\text { EN } \\
\text { PROCESOS }\end{array}$ & $\begin{array}{l}\text { ÁREA DE } \\
\text { RR.HH }\end{array}$ \\
\hline $\begin{array}{l}\text { Acuerdo } \\
\text { parcial }\end{array}$ & 139 & 92 & 73 & 100 & 106 \\
\hline $\begin{array}{l}\text { Acuerdo } \\
\text { total }\end{array}$ & 83 & 144 & 163 & 133 & 107 \\
\hline $\begin{array}{l}\text { Desacuerdo } \\
\text { parcial }\end{array}$ & 20 & 11 & 9 & 11 & 23 \\
\hline $\begin{array}{l}\text { Desacuerdo } \\
\text { total }\end{array}$ & 6 & 1 & 3 & 4 & 12 \\
\hline Total & 248 & 248 & 248 & 248 & 248 \\
\hline $\begin{array}{l}\text { Acuerdo } \\
\text { (total y } \\
\text { parcial) }\end{array}$ & $89,52 \%$ & $95,15 \%$ & $95,16 \%$ & $93,95 \%$ & $85,89 \%$ \\
\hline
\end{tabular}

De acuerdo con las respuestas, resalta el hecho de que el diagnóstico de necesidades y el refuerzo de resultados esperados sean dos de los elementos de base para diseñar un plan de capacitación. Le sigue en preferencia los cambios en los procesos y/o equipos/aplicativos informáticos. Consideran importante que el colaborador también pueda proponer temas en coordinación con los que el área de talento humano pueda establecer.

Finalmente, luego de procesar la información recogida, se obtuvieron insumos importantes para presentar una propuesta de temas que podrían ser incluidos en los futuros programas de capacitación en la UCSG. Estos fueron agrupados en tres ámbitos: institucionales, generales y específicos.

En cuanto a temas institucionales se pueden mencionar los siguientes:

- Alineamiento estratégico y compromiso institucional

- Clima laboral

- Comportamiento organizacional

- Cultura organizacional

- Desarrollo organizacional

- Estructura organizacional

- Ética profesional e institucional

- Imagen institucional

- Instructivos para manejo del Sistema Integrado Universitario (SIU)

- Manejo de la inducción y reinducción de las personas

- Manual de funciones, políticas, procesos y procedimientos

- Misión, visión y valores institucionales

- Normativas internas, estatuto

- Planeación estratégica y operativa

- Programas y proyectos institucionales
- Reglamentos de organismos de control gubernamental

- Responsabilidad social

- Sistema de información gerencial

- Transparencia de información, portal web

En lo relativo a temas generales para la formación personal y profesional, aplicables a todos los departamentos, y que pretenden desarrollar en el colaborador las competencias inherentes a su desempeño individual y grupal (en función del nivel jerárquico en el que se encuentre desempeñando sus funciones el colaborador), siempre con la orientación de la filosofía institucional, se proponen:

- Actitud positiva, base para una cultura de calidad

- Adaptación y flexibilidad ante el cambio

- Administración del tiempo

- Administración por procesos

- Aplicaciones informáticas y utilitarias

- Aprendizaje, autodesarrollo y efectividad

- Atención telefónica

- Atención y servicio al cliente interno y externo

- Autocontrol y tolerancia a la presión

- Autoestima, fuente de desarrollo personal

- Comunicación eficaz, asertiva

- Creatividad e innovación

- Desarrollo de habilidades del pensamiento

- Desarrollo gerencial

- Elaboración de informes

- Emprendimiento

- Finanzas para no financieros

- Gestión y prevención de riesgos laborales

- Inteligencia emocional

- Liderazgo ejecutivo

- Manejo del estrés

- Motivación

- Negociación y manejo de conflictos

- Organización y planificación del trabajo

- Orientación a resultados

- Pensamiento crítico, analítico y conceptual

- Programación neurolingüística

- Relaciones humanas

- Relaciones públicas

- Salud ocupacional

- Toma de decisiones 
- Trabajo colaborativo y cooperativo, trabajo en equipo

- Visión estratégica

Los siguientes temas específicos están relacionados con los diferentes procesos que se cumplen a lo interno de la institución, y que no son de un solo departamento, sino que se requiere la coordinación y continuidad entre departamentos.

- Actualización de la legislación laboral

- Administración de la cartera

- Administración de riesgos

- Archivología

- Balance scorecard

- Bases de datos

- Capacitación y desarrollo personal y profesional

- Conocimientos técnicos, según área de trabajo

- Contabilidad

- Control y sistemas de calidad

- Desarrollo de aplicaciones informáticas

- Desarrollo de competencias específicas

- Estrategia de negocios

- Facultamiento, delegación y liderazgo

- Gestión de bibliotecas

- Gestión de cobranzas

- Gestión de información estadística

- Gestión de procesos

- Gestión de proyectos

- Gestión del talento humano

- Integración de equipos de alto desempeño

- Mantenimiento de equipos

- Medición y control estadístico de procesos

- Mercado financiero

- Plan de carrera profesional

- Presupuesto

- Redes y cableado estructurado

- Seguridad y salud en el trabajo

- Técnicas de ventas

- Tributación e impuestos

- Ventas, publicidad, marketing

La frecuencia de aplicación de estos temas dependerá de las necesidades y exigencias que surgen continuamente en el quehacer diario de la universidad. La decisión final es responsabilidad de la Dirección de Recursos Humanos.

DISCUSIÓN

De acuerdo con los resultados obtenidos se puede apreciar que los colaboradores están conscientes de que el refuerzo de conocimientos actuales y la adquisición de nuevos conocimientos son básicos para su crecimiento profesional y personal, que redundará en un desempeño eficiente para beneficio institucional. Como complemento opinaron que serviría para lograr ascensos, estabilidad en el cargo y mejorar su empleabilidad. Con respecto a los temas a ser incluidos en el programa de capacitación, éstos deben ser diversos, básicos y específicos, que respondan a las necesidades laborales de la institución en general y de los puestos en particular, además de que incentive al colaborador en el desarrollo de la innovación y la creatividad, base para la solución de conflictos y la toma de decisiones.

En suma, la capacitación y desarrollo de las personas permite mejorar procesos, optimizar recursos y generar cambios de comportamiento orientados hacia la atención y servicio a los usuarios. Por tanto, de acuerdo con Martín Linares, Segredo Pérez y Perdomo Victoria (2013) se requiere identificar continuamente "las necesidades actuales y futuras en materia de conocimientos y experiencias, para gestionar de tal forma los recursos que cubran las necesidades identificadas, ya sea a corto, mediano o largo plazo"(p. 282), sobre todo en los tiempos actuales que a decir de Ramírez Ospina (2015) los avances en ciencia y tecnología priorizan la inversión en las personas para incrementar el capital humano.

CONCLUSIONES

Los temas propuestos no buscan superar en su totalidad cualquier insuficiencia en procesos, comportamientos, niveles de satisfacción y motivación con respecto a la tarea. El beneficio de un programa de capacitación debe verse reflejado en un resultado posterior al evento y siempre y cuando el colaborador haya participado con la intención de mejorar tanto en el ámbito personal como profesional. Los cambios deben ser dados por voluntad propia no por imposición, para que dé los frutos esperados.

El sentido de pertenencia hacia los objetivos y estrategias institucionales requiere un sustento en el respeto mutuo desde y hacia la persona, y desde y hacia la institución.

Las personas aprenden también con base en el ejemplo de su superior, subordinado y/o compañero de trabajo, dentro de un sistema de equidad y trato justo, de manera que todos los integrantes del equipo reciban la satisfacción de un trabajo bien hecho y de objetivos logrados. 


\section{REFERENCIAS BIBLIOGRÁFICAS}

Alles, M. A. (2009). Diccionario de preguntas: la trilogía. Buenos Aires: Granica.

Blanco Fernández, A. (2009). Desarrollo y evaluación de competencias en Educación Superior. Madrid: Narcea.

Chiang, M., Núñez, A., Martín, M. J., y Salazar, M. (2010). Compromiso del Trabajador hacia su Organización y la relación con el Clima Organizacional: Un Análisis de Género y Edad. Panorama socioeconómico, 28(40), 90-100.

Chiavenato, I. (2009). Gestión del talento humano (Tercera). México: McCraw-Hill/ Interamericana.

Chiavenato, I. (2011). Administración de recursos humanos: el capital humano de las organizaciones. México D.F.: McGraw-Hill.

Dolan, S. L., Valle Cabrera, R., Jackson, S. E., y Schuler, R. S. (2007). La gestión de los recursos humanos: cómo atraer, retener y desarrollar con éxito el capital humano en tiempos de transformación (Tercera). Madrid: McGraw-Hill, Interamericana de España.

Gallego, D. J., y Gil, A. J. (2012). La construcción de organizaciones de aprendizaje a través del liderazgo. Universidad \& Empresa, 14(22), 43-78.
Gil López, A. J., y Carrillo Gamboa, F. J. (2013). Knowledge creation in organizations through learning. Intangible Capital, 9(3). https://doi.org/10.3926/ic.418

González Bertolín, A. (2011). El aprendizaje por competencias en la educación obligatoria. Valencia: Brief.

Ibarra Almada, A. (2000). Formación de los recursos humanos y competencia laboral. Boletín CINTEFOR, 149, 95-108.

Martín Linares, X., Segredo Pérez, A. M., y Perdomo Victoria, I. (2013). Capital humano, gestión académica y desarrollo organizacional. Educación Médica Superior, 27(3), 288-295.

Nonaka, I. (2007). La empresa creadora de conocimiento. Chile: Harvard Business Review América Latina.

Ramírez Ospina, D. E. (2015). Capital humano: una visión desde la teoría crítica. Cadernos EBAPE.BR, 13(2), 315-331. https://doi. org/10.1590/1679-395114754

Robbins, S. P., y Coulter, M. K. (2014). Administración (Décimo Segunda Edición). México, D.F.: Pearson.

Schermerhorn, J. R. (2010). Administración (Segunda). México: Limusa. 